\title{
Distribución de biomasa aérea en un bosque de Pinus patula bajo gestión forestal en Zacualtipán, Hidalgo, México
}

\section{Aboveground biomass allocation in a managed forest of Pinus patula in Zacualtipán, Hidalgo, Mexico}

\author{
Griselda Chávez-Aguilarl, Gregorio Ángeles-Pérez*, Marlín Pérez-Suárez², Miguel Ángel López-López', \\ Edmundo García-Moya ${ }^{3}$ y Craig Wayson ${ }^{4}$
}

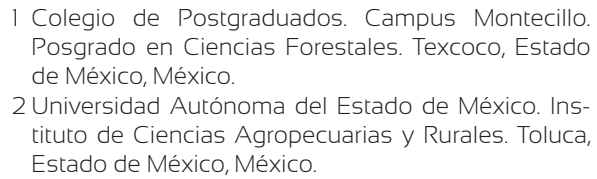
Posgrado en Ciencias Forestales. Texcoco, Estado de México, México.

2 Universidad Autónoma del Estado de México. Instituto de Ciencias Agropecuarias y Rurales. Toluca, Estado de México, México.

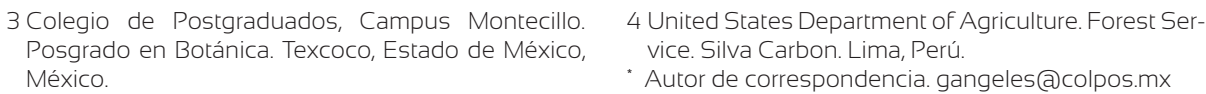

4 United States Department of Agriculture. Forest Service. Silva Carbon. Lima, Perú.

Autor de correspondencia. gangeles(a)colpos.mx

\section{RESUMEN}

La acumulación y distribución de biomasa hacia los componentes estructurales de los árboles es determinante en bosques manejados, tanto en términos de productividad maderable como en la reactivación de diversos procesos ecosistémicos. En el presente estudio se analizó el patrón de acumulación de biomasa aérea total (BAT) y su asignación hacia fuste, ramas, corteza y follaje en una cronosecuencia de un bosque de Pinus patula bajo aprovechamiento forestal en Zacualtipán, Hidalgo. Mediante el uso de ecuaciones alométricas se estimó la BAT y por componente estructural de los árboles en rodales con diferentes años después de la cosecha, en diferentes años de remedición (años 2005, 2008 y 2012). Se encontró que la BAT aumentó con el tiempo después de la cosecha, con diferencias estadísticas significativas $(\mathrm{p}<0.0001)$ entre los rodales. El rodal de 30 años después de la cosecha presentó una biomasa aérea total (BAT) de 178.1 $\mathrm{Mg} \mathrm{ha}^{-1}$, la cual resultó solo $20 \%$ menor a la observada en el rodal sin cosecha (AN), lo que indica que un bosque bajo aprovechamiento podría alcanzar niveles de biomasa aérea similares a la del AN, en un menor tiempo. La asignación de biomasa aérea (BA) presentó el siguiente orden: fuste > ramas> corteza > follaje. Esta asignación de BA fue favorecida hacia fuste como un producto maderable comercial, objetivo principal de estos bosques bajo producción. El patrón de asignación de BA entre los componentes estructurales de los árboles puede ser incorporado como base para el diseño de prácticas silvicuturales en los programas de manejo forestal y contribuir en la evaluación de la sustentabilidad de los bosques. Además, puede clarificar el papel de los bosques regenerados después de los aprovechamientos en la fijación y almacenamiento de bióxido de carbono atmosférico.

PALABRAS ClAVE: componente estructural, follaje, fuste, prácticas silviculturales, ramas, rodal.

\section{ABSTRACT}

Aboveground biomass accumulation and allocation to the structural components of trees is crucial in managed forests, both in terms of timber productivity and the reactivation of different ecosystem processes. In this study, we analyzed the pattern of accumulation of aboveground total biomass and its allocation among stems, branches, bark and foliage in a chronosequence of managed Pinus patula forests in Zacualtipán, Hidalgo. Total aboveground biomass (TAB) and its allocation to structural components were estimated by using allometric equations in stands with different numbers of years since harvest, in different years of measurement (2005, 2008 and 2012). We found that the TAB increased with time since harvest, with highly significant differences $(\mathrm{p}<0.0001)$ among stand ages. The stand with 30 years since harvest showed an aboveground biomass (AB) of $178.1 \mathrm{Mg} \mathrm{ha}^{-1}$ which was only $20 \%$ lower than that estimated for the stand without harvest (AN), indicating that a managed forest could reach levels of aboveground biomass similar to those of the AN, but in less time. Aboveground biomass allocation was made in the following order: stem> branches $>$ bark $>$ foliage. Allocation favored the stem as a commercial timber product, which is the main objective of these managed forests. The pattern of aboveground biomass allocation among the structural components of the trees reflects the fact that forest management in temperate forests operates as an important alternative for the recovery of degraded forests. Because of climate change, it can also represent an appropriate strategy for carbon sequestration and mitigation of carbon dioxide emissions.

KEY WORDS: structural components, foliage, stem, silvicultural practices, branches, stand. 


\section{INTRODUCCIÓN}

La gestión forestal sostenible representa en muchas regiones del mundo una alternativa útil para la reducción de la deforestación y degradación de los ecosistemas forestales (von Gadow, Sánchez y Aguirre, 2004; Durán, Mas y Velázquez, 2007; Galicia, Saynes y Campo, 2015). La gestión de los bosques permite su conservación y la producción de bienes y servicios demandados por la sociedad; lo que constituye una estrategia apropiada para la fijación y reducción de las emisiones de bióxido de carbono y, por tanto, la mitigación del cambio climático (Pompa-García y Yerena-Yamalliel, 2014; Torres-Rojo, Moreno-Sánchez y Mendoza-Briseño, 2016).

A través de la gestión forestal sostenible se busca principalmente el aumento en la producción maderable como resultado del incremento del volumen fustal, mediante prácticas silviculturales de aplicación intensiva (Jandl et al., 2007; Porter-Bolland et al., 2012). Lo anterior trae como consecuencia el aumento de la biomasa forestal y su cuantificación es de suma importancia para entender el funcionamiento y dinámica de los ecosistemas forestales, y además porque es una de las principales formas de cuantificar la capacidad de fijación de carbono en la vegetación (Montero, Ruiz-Peinado y Muñoz, 2005; Brandeis, Delaney, Parresol y Royer, 2006) que a su vez, es uno de los factores determinantes en la sustentabilidad de los bosques (Montero et al., 2005). De esta forma, la gestión forestal sostenible de ecosistemas terrestres comprende la búsqueda de conocimiento sobre los bienes y servicios del ecosistema, propiciando la necesidad de cuantificar no únicamente la biomasa total de los árboles, sino su distribución hacia los diferentes componentes estructurales (Bazzaz, 1997; Montero et al., 2005).

La distribución de la biomasa varía en cada componente estructural del árbol y depende de la función que desempeña cada uno dentro del ecosistema (Bazzas, 1997). Por ejemplo, la asignación de biomasa hacia fuste influye sobre la producción de madera aprovechable, pero también es fundamental para la conducción de nutrientes y agua (von Gadow et al., 2004), mientras que la asignación hacia ramas, influye sobre la capacidad de soporte de follaje. Por su parte, hacia el follaje influye sobre la fotosíntesis y las tasas de reincorporación de nutrimentos hacia el piso forestal. De esta forma, los cambios en el almacenamiento y distribución de la biomasa pueden también estar fuertemente influenciados por factores relacionados con factores como: desarrollo del rodal, edad, calidad del sitio, composición de especies, variables ambientales, densidad del rodal, prácticas silvícolas aplicadas (Figueroa, Ángeles, Velázquez y de los Santos, 2010; Rodríguez-Ortíz et al., 2012; Soriano-Luna, ÁngelesPérez, Martínez-Trinidad, Plascencia-Escalante y RazoZárate, 2015), entre otros.

Paralelamente, la estimación de la distribución de dicha biomasa viene acompañada con el conocimiento de procesos de suma importancia para el ecosistema, tales como productividad, ciclo de nutrientes, movimiento de materia y energía, fijación y almacenamiento de carbono (Castillo, Imbert, Blanco, Traver y Puertas, 2003; Escobar et al., 2008; Gerez-Fernández y Pineda-López, 2011; León, González y Gallardo, 2011; Pompa-García y Yerena-Yamalliel, 2014; Aquino-Ramírez, VelázquezMartínez, Castellanos-Bolaños, De los Santos-Posadas y Etchevers-Barra, 2015) y en la evaluación de la gestión forestal sostenible (Brandeis et al., 2006) cuando de bosques intervenidos se trata. Sin embargo, seguir el patrón de cambios en esta distribución de biomasa a través del tiempo requiere de instrumentación de estudio a largo plazo. Por lo tanto, una forma de superar esta limitante es la utilización del enfoque de cronosecuencia, donde se sustituye espacio por tiempo (Law, Sun, Campbell, Van Tuyl y Thornton, 2003). En bosques bajo gestión forestal este enfoque representa una herramienta útil que permite comparar la estructura y atributos funcionales del ecosistema en intervalos de condiciones ambientales similares (Law et al., 2003; Figueroa et al., 2010).

Los estudios para la cuantificación de la biomasa aérea para conocer su asignación en los diversos componentes estructurales de los árboles en especies forestales son frecuentes (Acosta-Mireles, Vargas-Hernández, Velázquez-Martínez y Etchevers-Barra, 2002; GómezDíaz, Etchevers-Barra, Monterrosos-Rivas, Campo-Alvez 
y Tinoco-Rueda, 2011; Díaz-Franco et al., 2007; Figueroa et al., 2010; Soriano-Luna et al., 2015), sin embargo, la mayoría de ellos se han realizado a nivel de individuo y han estado encaminados a la generación de funciones matemáticas para la estimación de biomasa total y de carbono (Pompa-García y Yerena-Yamalliel, 2014; AquinoRamírez et al., 2015), o bien, para el mejoramiento genético y establecimiento de plantaciones forestales (Morales-González, López-Upton, Vargas-Hernández, Ramírez-Herrera y Muñoz, 2013). La región de Zacualtipán, Hidalgo, México, es un ejemplo de ello, donde se han realizado estudios significativos para la cuantificación de la biomasa aérea forestal en bosques de Pinus patula bajo aprovechamiento (Aguirre-Salado et al., 2009; Figueroa et al., 2010; Soriano-Luna et al., 2015). Pese a ello, se carece de información sobre el patrón de cambios en la distribución de dicha biomasa en los diferentes componentes estructurales de los árboles relacionados directamente con el desarrollo del rodal.

\section{OBjetivos}

Cuantificar la biomasa aérea total y determinar el patrón de su distribución en los diferentes componentes estructurales de los árboles: fuste, ramas, corteza y follaje, a lo largo del desarrollo estructural de rodales en un bosque de $P$. patula bajo aprovechamiento forestal. Así como realizar una comparación de dicha biomasa con aquella registrada en un área sin intervención silvícola, bajo la hipótesis de que no hay diferencias en la capacidad de acumulación de biomasa aérea entre ambas condiciones.

\section{MATERIALES Y MÉTODOS}

\section{Área de estudio}

El área de estudio se localiza en el ejido La Mojonera, al sureste del municipio de Zacualtipán de Ángeles en el estado de Hidalgo a 20³7'11' y 20³7'43" N y 98 36'22" y 98 37’37’ O (Fig. 1). Se encuentra en la provincia fisiográfica de la Sierra Madre Oriental, conocida como Sierra Alta Hidalguense y una parte de la Faja Volcánica Transmexicana (Eje Neovolcánico), subprovincia Carso
Huasteco. Está conformado por pendientes, mesetas y cañones, con una altitud media de $2100 \mathrm{~m}$. El relieve es generalmente accidentado con pendientes de $0 \%$ a $25 \%$ y la exposición dominante es Norte y Noroeste. El clima que predomina es templado húmedo $[\mathrm{C}(\mathrm{m})]$ y templado subhúmedo $[\mathrm{C}(\mathrm{w} 2)]$ con temperatura media anual entre $12{ }^{\circ} \mathrm{C}$ y $18{ }^{\circ} \mathrm{C}$. La temperatura del mes más frío puede llegar a $-3{ }^{\circ} \mathrm{C}$ y el promedio en el mes más cálido a $22^{\circ} \mathrm{C}$. La precipitación media anual oscila entre $700 \mathrm{~mm}$ y 2050 mm y la precipitación del mes más seco puede llegar a 40 $\mathrm{mm}$. Se presentan lluvias casi todo el año (García, 2004) y las lluvias de verano e invierno pueden comprender $5 \%$ a $10.2 \%$ de la total anual (Servicio Meteorológico Nacional, 2014). El período de lluvias es de junio a septiembre, aunque se presenta un período de sequía aproximadamente entre febrero y mayo. La zona presenta abundante
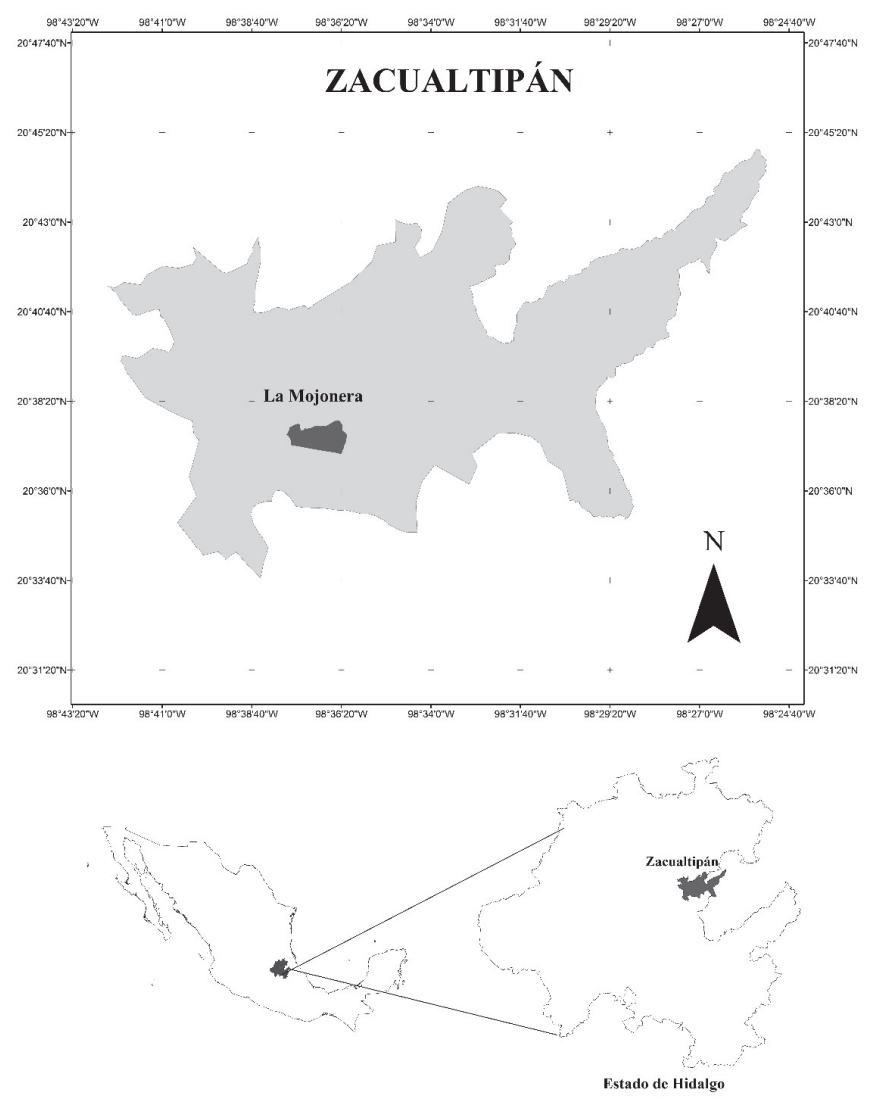

Figura 1. Ubicación geográfica del ejido La Mojonera en Zacualtipán Hidalgo, México. 
pedregosidad y el sustrato geológico data del período Cenozoico (terciario superior) conformado por rocas ígneas extrusivas del tipo toba ácida (Ts-Ta al Norte) y basalto (Ts-B al Sur). La unidad geológica Ts (Ta) está representada por tobas e ignebritas de composición riolítica y dacítica asociadas con intercalaciones gruesas de obsidiana y derrames basálticos y riolíticos. El suelo predominante es feozem háplico y secundariamente el regosol calcárico y litosol, con buen drenaje, textura media y alto contenido de materia orgánica y nutrimentos. Los principales tipos de vegetación a nivel regional son bosque de encino, pino-encino y bosque mesófilo de montaña (Ponce-Vargas, Luna-Vega, Alcántara-Ayala y RuizJiménez, 2006), que es un ecosistema que alberga un gran número de especies vegetales de restringida distribución natural y que es altamente vulnerable por la alta presión antropógena que sufre actualmente (Rzedowski, 1996).

El aprovechamiento de los recursos forestales y no forestales en el ejido se originó a consecuencia de la deforestación y el cambio de uso de la tierra en la región (Ángeles-Pérez, 2009). El ejido está constituido por 316 ha en total, de las cuales aproximadamente 160 ha se encuentran con aprovechamiento maderable a partir de 1982 mediante el método de desarrollo silvícola (MDS), en el cual se utiliza el método de repoblación de árboles padres (Castelán-Lorenzo y Arteaga-Martínez, 2009; TorresRojo et al., 2016). Este método produce rodales coetáneos donde la especie de mayor distribución e importancia económica es P. patula (Patiño y Kageyama, 1992; Dvorak, 2000; Velázquez, Ángeles, Llanderal, Román y Reyes, 2004; Castelán-Lorenzo y Arteaga-Martínez, 2009). Todos los rodales recibieron las mismas prácticas silviculturales; además de la corta de repoblación por árboles padres, se aplican cortas intermedias como preparación del sitio, limpias, cortas de liberación, aclareos, podas, y cortas de mejoramiento y saneamiento.

En los rodales intervenidos la especie que domina es $P$. patula y en menor abundancia se pueden encontrar $P$. teocote Schltdl. \& Cham. En áreas de vegetación natural se ecuentran especies como Quercus excelsa Liebm., Q. laurina Hump et Bonpl, Q. rugosa Neé, Q. castanea Neé,
Clethra mexicana D.C., Cleyera theaoides Sw., Vaccinium leucanthum Schltdl., Cornus disciflora Moc. \& Sessé ex D.C., Alnus sp., Arbutus jalapensis Kunth., Prunus serótina Ehrh., entre otras (Ángeles-Pérez, 2009; Figueroa et al., 2010) que también están asociadas a la vegetación en rodales con aprovechamiento.

\section{Establecimiento de las unidades de muestreo y medición de variables dasométricas}

Se seleccionaron rodales con las siguientes anualidades: 1998, 1995, 1991, 1988, 1984 y 1982. Cada anualidad correspondió al año en que fue aplicada la corta de repoblación. Adicionalmente, se seleccionó un bosque sin intervención silvícola de aproximadamente 80 años de edad, utilizado en el presente estudio como área de referencia (AN). En cada rodal y AN se establecieron al azar tres unidades de muestreo permanentes (UMP), cada una de $400 \mathrm{~m}^{2}$ en el año 2005. Las UMP permiten evaluar el comportamiento de la asignación de biomasa aérea del bosque a través del tiempo (variación temporal). Dentro de cada UMP se identificaron y etiquetaron todos los individuos arbóreos con una altura $\geq 1.3 \mathrm{~m}$ y diámetro normal $\geq 2.5 \mathrm{~cm}$. Estas variables dasométricas fueron documentadas en los mismos individuos en los años 2005, 2008 y 2012; en el caso del AN se realizaron dos mediciones en 2005 y 2012. Las diferencias en las características estructurales de los rodales tuvieron relación con sus edades al momento de las remediciones, y la edad del rodal se definió como el tiempo transcurrido a partir del año en que se aplicó la corta de repoblación. Es importante mencionar que la comparación entre edades a nivel de individuo no formó parte de los objetivos del presente estudio.

\section{Estimación de la biomasa aérea total y por componente estructural}

La biomasa aérea total (BAT) y por componente estructural (fuste, ramas, corteza y follaje) fue estimada con los modelos de biomasa generados para la misma área de estudio (Soriano-Luna et al., 2015). La BAT incluyó diferentes especies de latifoliadas (Q. excelsa, Q. laurina, Q. 
rugosa, Clethra mexicana, Cornus disciflora, Arbutus jalapensis, Vaccinium leucanthum) y P. patula. El modelo de biomasa presentó la siguiente forma:

$B=\operatorname{Exp}\left(-\beta_{0}\right) *\left(d n^{2} * h\right)^{\beta 1}$

Donde:

$B: \quad$ biomasa por árbol

$d n: \quad$ diámetro normal (DAP)

$h$ : $\quad$ altura total del árbol

$\beta_{0} \mathrm{y} \beta_{1}$ : estimadores cuyos valores se presentan en la tabla 1

La mayoría de los modelos matemáticos utilizan el DAP como variable estimadora de la biomasa (Vásquez y Arellano, 2012), lo cual podría conducir a errores estadísticos en la estimación de la biomasa aérea total (Chave et

Tabla 1. Estimadores estadísticos obtenidos del modelo alométrico por tipo de especie, en el cálculo de la biomasa aérea total y por componente estructural.

\begin{tabular}{ccc}
\hline Parámetro & Pinus patula & Spp. Latifoliadas \\
\hline Biomasa total: & & \\
$\beta_{0}$ & 4.554805 & 3.109407 \\
$\beta_{1}$ & 1.047218 & 0.952688 \\
Fuste: & & \\
$\beta_{0}$ & 4.682959 & 4.196867 \\
$\beta_{1}$ & 1.033543 & 0.988965 \\
Ramas: & & \\
$\beta_{0}$ & 5.510841 & 6.663739 \\
$\beta_{1}$ & 0.951067 & 1.208846 \\
Corteza: & & \\
$\beta_{0}$ & 7.441298 & 5.630984 \\
$\beta_{1}$ & 1.103910 & 0.949278 \\
Follaje: & & \\
$\beta_{0}$ & 4.750974 & 2.437957 \\
$\beta_{1}$ & 0.709796 & 0.574860 \\
\hline
\end{tabular}

Fuente: Soriano-Luna et al. (2015). al., 2004). Sin embargo, para tratar de reducir algunos de esos errores el modelo utilizado en este estudio estimó la biomasa en función del diámetro (DAP) con la inclusión de la altura del árbol $(\mathrm{H})$, lo cual reduce el error estándar, mejora el ajuste de los modelos matemáticos (SorianoLuna et al., 2015) y, dado que estos parámetros son responsables de la variación de la biomasa, proporcionan información indirecta sobre las características del entorno competitivo como la edad del rodal, índice de sitio, densidad, etcétera (Vásquez y Arellano, 2012; Soriano-Luna et al., 2015).

\section{Análisis estadísticos}

Los análisis estadísticos se llevaron a cabo utilizando como repeticiones a las observaciones realizadas dentro de cada rodal que constituyeron pseudoréplicas (Pérez, Frangi, Goya, Luy y Arturi, 2013). En consecuencia, se evaluaron las diferencias entre los rodales. La BAT se comparó entre rodales mediante un análisis de varianza (Anova) de una vía usando como factor principal el rodal. Se realizó un análisis a posteriori de las diferencias entre rodales mediante la prueba de Tukey HSD $(\alpha \leq 0.05)$ con el paquete estadístico SAS (2009). La proporción relativa para cada componente se estimó a partir de la biomasa total, con la finalidad de comparar la asignación de biomasa aérea entre los rodales. Esto se realizó para cada año de registro (2005, 2008 y 2012).

\section{RESULTADOS}

\section{Biomasa aérea total}

La biomasa aérea total (BAT) presentó diferencias estadísticas altamente significativas $(\mathrm{p}<0.0001$; Tabla 2$)$ entre rodales (Fig. 2) y aumentó a lo largo de la cronosecuencia del bosque de $P$. patula bajo aprovechamiento forestal. Sin embargo, una excepción a esta tendencia fue observada en el rodal de la anualidad 1995 en la remedición 2005 (Fig. 2a) que mostró $10 \%$ menor BAT que el rodal de anualidad 1998 (Fig. 2a), dicho comportamiento coincidió con que en el primer rodal (1995) la densidad fue dos veces mayor que la del segundo (Tabla 3). En la remedición 2008 se 
TABLA 2. Resultados del análisis de varianza (Anova) para la biomasa aérea total en rodales de diferente edad de aprovechamiento, remedidos en tres años diferentes (2005, 2008 y 2012).

\begin{tabular}{cccccc}
\hline $\begin{array}{c}\text { Año de } \\
\text { remedición }\end{array}$ & SC & CM & GL & $F_{0}$ & Pr>F \\
\hline 2005 & 71779.26 & 11963.21 & 6 & 18.94 & $<0.0001$ \\
2008 & 23117.98 & 4623.59 & 5 & 16.93 & \\
2012 & 44811.7 & 7468.61 & 6 & 22.11 & \\
\hline
\end{tabular}

Donde; $\mathrm{SC}=$ Suma de cuadrados, $\mathrm{CM}=$ Cuadrado de la media, $\mathrm{GL}=$ Grados de libertad, $\mathrm{F}_{0}=$ Estadístico $\mathrm{F}$ de Fisher y $\mathrm{Pr}=$ Probalidad

Tabla 3. Área basal y densidad en rodales de diferente edad de aprovechamiento, durante un período de siete años (2005-2012) en un bosque de Pinus patula bajo gestión forestal en Zacualtipán Hidalgo.

\begin{tabular}{ccccccc}
\hline \multirow{2}{*}{ Anualidad } & \multicolumn{3}{c}{ Área basal $\left(\mathrm{m}^{2} \mathrm{ha}^{-1}\right)$} & \multicolumn{3}{c}{ Densidad (árboles ha-1) } \\
\cline { 2 - 7 } & 2005 & 2008 & 2012 & 2005 & 2008 & 2012 \\
\hline 1998 & 21.3 & 35.7 & 25.7 & 4692 & 4100 & 1708 \\
1995 & 14.8 & 27.5 & 34.5 & 2292 & 2208 & 1917 \\
1991 & 17.9 & 23.5 & 27.9 & 733 & 733 & 700 \\
1988 & 24.5 & 30.4 & 31.4 & 1133 & 1100 & 592 \\
1984 & 32.6 & 31.4 & 34.9 & 2308 & 1908 & 1808 \\
1982 & 36.1 & 39.7 & 39.9 & 4083 & 3908 & 3583 \\
AN & 36.7 &.$*$ & 38.2 & 2150 &. & 1867 \\
\hline " AN = área sin aprovechamiento forestal de aproximadamente ochenta años \\
de edad. \\
"* Los puntos en las celdas indican que no se realizó registro en ese año.
\end{tabular}

presentó un comportamiento similar (Fig. 2b), lo cual se debió a la aplicación de aclareos. Este efecto fue observado en el rodal de anualidad 1998 con una disminución

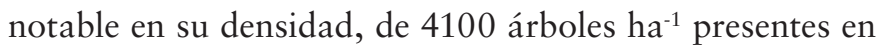
la remedición 2008 a 1708 árboles ha-1 en la remedición 2012 (Tabla 3), lo cual representó más de la mitad de extracción en términos de densidad (59\%). Así mismo, el rodal de anualidad 1988 mostró una disminución en su densidad que fue de 1100 árboles ha ${ }^{-1}$ cuantificados en la remedición 2008 a 592 árboles ha-1 en la remedición 2012, representando aproximadamente $46 \%$ de remoción de árboles (Tabla 3). Por su parte, la variación temporal de la densidad de árboles fue menor en etapas más avanzadas de desarrollo de los rodales; se observó que en el rodal de anualidad 1984 y 1982 representaron solamente 5\% y 8\% de disminución de la densidad (Tabla 3).

Cabe destacar que, pese a estos altos porcentajes de extracción, al finalizar el período de medición en el presente estudio la biomasa aérea total no resultó afectada, ya que los rodales tuvieron el tiempo para recuperar e incluso aumentar la biomasa removida por los aclareos. Ejemplo de lo anterior, se observó en el rodal 1995 que de $30.51 \mathrm{Mg} \mathrm{ha}^{-1}$ en la remedición 2005 aumentó a $99.95 \mathrm{Mg}$ ha $^{-1}$ en la remedición 2012, es decir, aumentó 70\% de su BAT (Fig. 2a y c). Por su parte, el rodal de mayor desarrollo estructural (anualidad 1982) para la remedición 2012 (Fig. 2c) presentó $78 \%$ (178.1 $\left.\mathrm{Mg} \mathrm{ha}^{-1}\right)$ de la BAT cuantificada en el AN (227.6 $\left.\mathrm{Mg} \mathrm{ha}^{-1}\right)$, sin diferencias estadísticamente significativas ( $<<0.0001$; Tabla 2) entre ellos (Fig. 2c). Esto indicó que el contenido de BAT fue $22 \%$ menor que la registrada en $\mathrm{AN}$, lo cual ocurrió 30 años después de haber sido aplicada la corta de repoblación.

Durante las primeras etapas de desarrollo de los rodales (anualidad 1998, 1995 y 1991), la BAT no fue estadísticamente diferente entre ellos $(\mathrm{p}<0.0001$; Tabla 2); el mismo patrón se presentó en las tres remediciones (Fig. 2). Sin embargo, la BAT fue diferenciándose a partir de etapas de desarrollo intermedias, tal como se observó en los rodales de anualidad 1988, 1984 y 1982 (Fig. 2a, b y c) en un intervalo de 17 a 30 años después de haber aplicado la corta de repoblación.

\section{Biomasa aérea por componente estructural}

La distribución de la biomasa aérea (BA) en los diferentes componentes estructurales de los árboles a lo largo del desarrollo estructural de los rodales presentó el siguiente orden descendente general: fuste> ramas $>$ corteza $>$ follaje (Tabla 4). Así mismo, el contenido de BA para todos los componentes aumentó con el desarrollo del rodal, excepto 

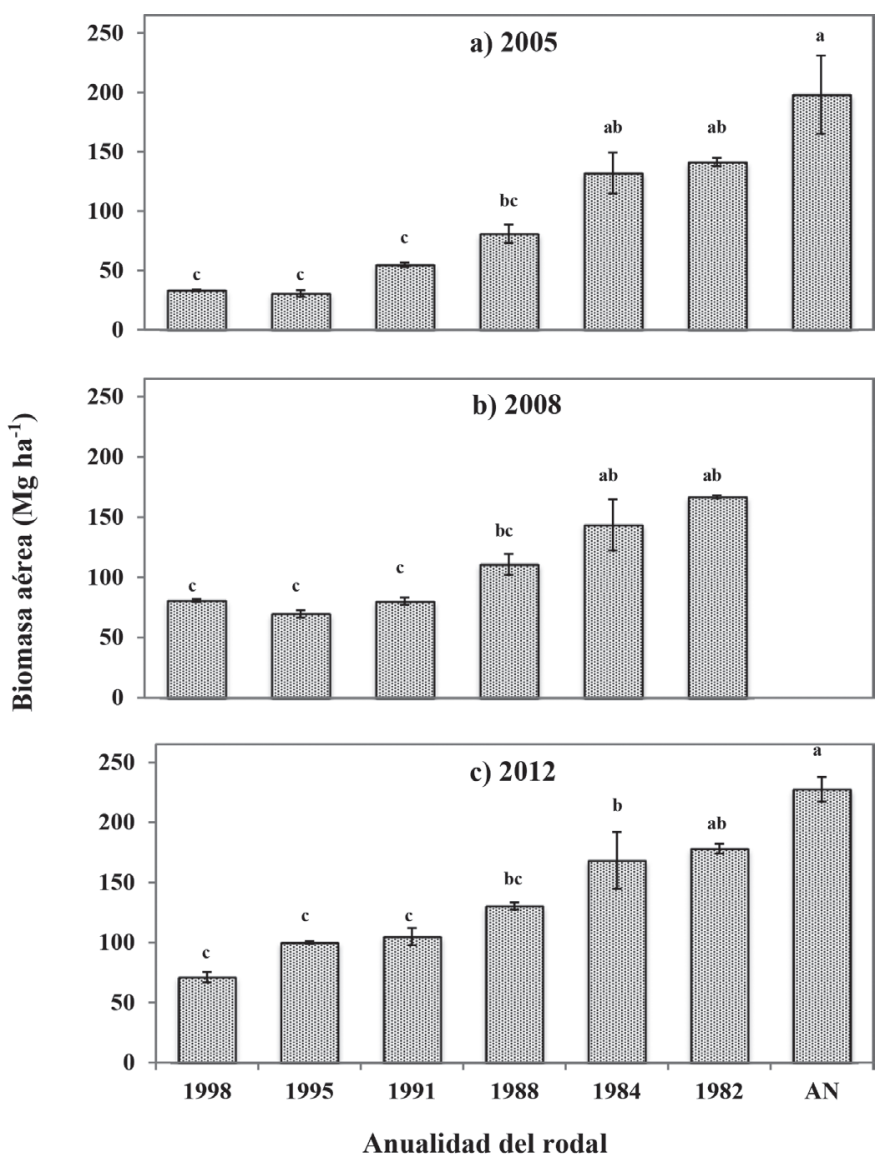

Figura 2. Biomasa aérea total $\left(\mathrm{Mg} \mathrm{ha}^{-1}\right)$ en rodales de diferente edad, durante un período de siete años (2005-2012), en un bosque de Pinus patula bajo gestión forestal en Zacualtipán Hidalgo. AN es un área sin aprovechamiento forestal de aproximadamente 80 años de edad.

para el rodal de anualidad 1998 que mostró aproximadamente 1.5 veces mayor BA que el rodal de anualidad 1995 (Tabla 3). Por su parte, únicamente en el rodal de anualidad 1995 se observó una disminución en la remedición 2012 con respecto a la BA registrada en la del 2008 (Tabla 4).

El fuste fue el componente estructural con mayor contenido y asignación respecto a la BAT (Tabla 4 y Fig. 3). Este componente presentó una BA de $25 \mathrm{Mg} \mathrm{ha}^{-1}$ en el rodal de anualidad 1998 y representó una quinta parte de la contenida en el AN. Mientras, que el rodal con mayor desarrollo (anualidad 1982) mostró una BA de $125 \mathrm{Mg}$ ha ${ }^{-1}$ en fuste, que fue $20 \%$ menor en comparación a la obtenida en el AN (Tabla 4). Durante las primeras etapas de desarrollo de los rodales (anualidad 1998, 1995 y 1991) la asignación de BA en fuste representó $69 \%$ a $72 \%$ de la BAT (Fig. 3). A medida que los rodales avanzaban en edad y desarrollo, la asignación de BA hacia fuste se mantuvo relativamente constante, entre $70 \%$ y $71 \%$ de la BAT (Fig. 3). En el AN se encontró que la asignación de BA hacia fuste disminuyó hasta $66 \%$ de la BAT en comparación con el resto de los rodales (Fig. 3a y c).

Las ramas fueron el segundo componente estructural con la mayor asignación de BA (Tabla 4), la cual disminuyó con el aumento en edad de aprovechamiento y desa-

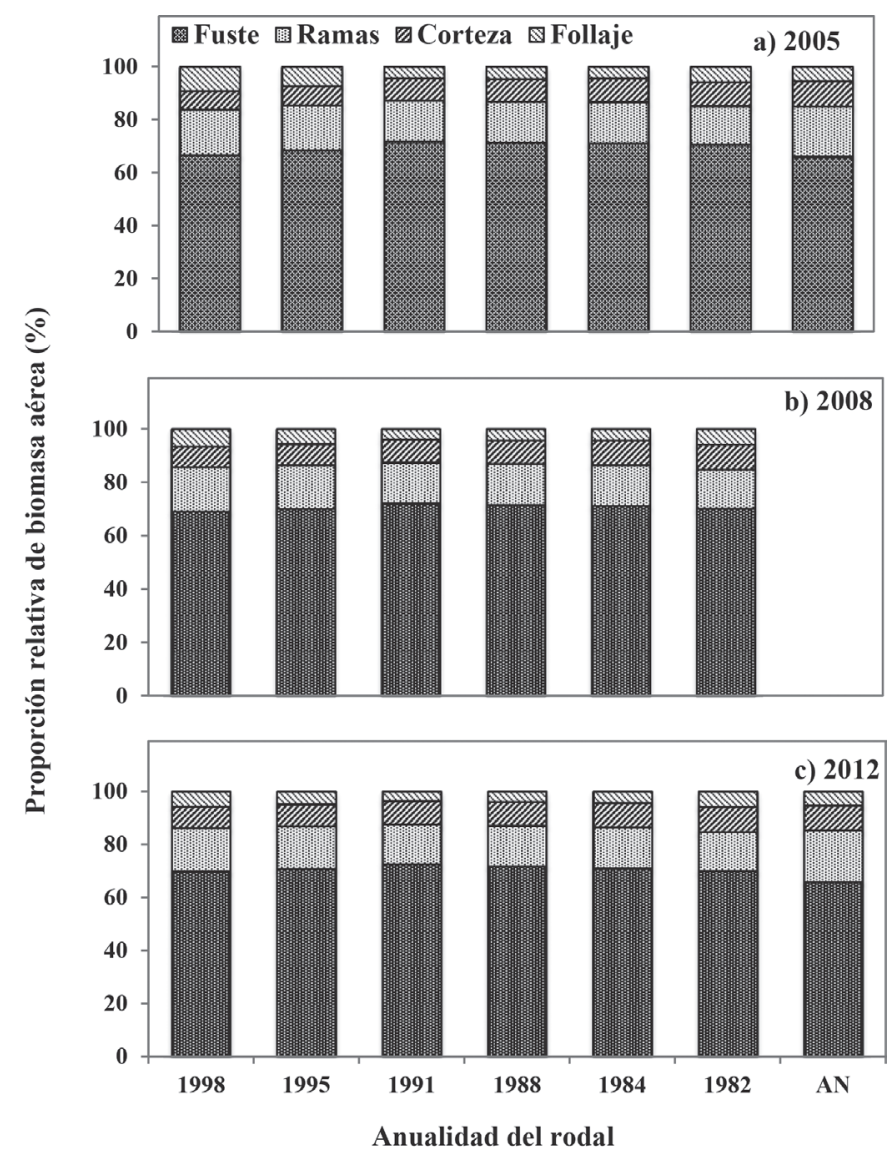

Figura 3. Distribución de biomasa aérea (\%) por componente estructural en un bosque de Pinus patula bajo gestión forestal en Zacualtipán Hidalgo, en diferentes años de registro. AN es un área sin aprovechamiento forestal de aproximadamente 80 años de edad. 
Tabla 4. Biomasa aérea $\left(\mathrm{Mg} \mathrm{ha}^{-1}\right)$ por componente en rodales de diferente edad, durante un período de siete años (2005-2012), en un bosque de Pinus patula bajo gestión forestal en Zacualtipán Hidalgo.

\begin{tabular}{|c|c|c|c|c|}
\hline Anualidad & Componente & 2005 & 2008 & 2012 \\
\hline \multirow[t]{5}{*}{1998} & Fuste & 25.65 & 62.38 & 53.68 \\
\hline & Ramas & 6.65 & 15.04 & 12.62 \\
\hline & Corteza & 2.66 & 6.84 & 6.21 \\
\hline & Follaje & 3.55 & 6.03 & 4.38 \\
\hline & Total $^{*}$ & 38.50 & 90.30 & 76.88 \\
\hline \multirow[t]{5}{*}{1995} & Fuste & 23.71 & 53.88 & 76.79 \\
\hline & Ramas & 5.88 & 12.61 & 17.37 \\
\hline & Corteza & 2.54 & 6.06 & 8.90 \\
\hline & Follaje & 2.50 & 4.34 & 5.30 \\
\hline & Total & 34.63 & 76.89 & 108.37 \\
\hline \multirow[t]{5}{*}{1991} & Fuste & 42.49 & 61.82 & .57 \\
\hline & Ramas & 9.20 & 13.09 & 16.68 \\
\hline & Corteza & 4.96 & 7.40 & 9.85 \\
\hline & Follaje & 2.56 & 3.36 & 3.96 \\
\hline & Total & 59.21 & 85.67 & 111.07 \\
\hline \multirow[t]{5}{*}{1988} & Fuste & 61.40 & 83.45 & 97.23 \\
\hline & Ramas & 13.34 & 17.91 & 20.64 \\
\hline & Corteza & 7.37 & 10.24 & 12.27 \\
\hline & Follaje & 4.03 & 5.01 & 5.28 \\
\hline & Total & 86.14 & 116.61 & 135.43 \\
\hline \multirow[t]{5}{*}{1984} & Fuste & 98.28 & 105.06 & 122.13 \\
\hline & Ramas & 21.33 & 22.63 & 26.54 \\
\hline & Corteza & 12.43 & 13.72 & 16.17 \\
\hline & Follaje & 6.07 & 6.37 & 7.25 \\
\hline & Total & 138.10 & 147.78 & 172.09 \\
\hline \multirow[t]{5}{*}{1982} & Fuste & 103.21 & 119.18 & 125.58 \\
\hline & Ramas & 21.18 & 24.86 & 26.45 \\
\hline & Corteza & 13.26 & 15.69 & 16.83 \\
\hline & Follaje & 8.43 & 10.06 & 10.47 \\
\hline & Total & 146.08 & 169.80 & 179.34 \\
\hline \multirow[t]{5}{*}{$\mathrm{AN}^{* *}$} & Fuste & 127.55 &.$^{* * *}$ & 146.96 \\
\hline & Ramas & 36.78 & . & 43.39 \\
\hline & Corteza & 18.35 & . & 20.98 \\
\hline & Follaje & 10.39 & . & 11.65 \\
\hline & Total & 193.06 & . & 222.98 \\
\hline
\end{tabular}

* Biomasa aérea total estimada como la suma de la estimación por componente.

* $\mathrm{AN}$ = área sin aprovechamiento forestal de aproximadamente 80 años de edad.

*** Los puntos en las celdas indican que no se realizó medición en ese año. rrollo del rodal. En las últimas etapas de desarrollo evaluadas, representadas en los rodales de anualidad 1984 y 1982, el contenido de BA se mantuvo relativamente constante $\left(21.33 \mathrm{Mg} \mathrm{ha}^{-1}\right.$ y $21.18 \mathrm{Mg} \mathrm{ha}^{-1}$, respectivamente). Mientras que en el AN se observó mayor contenido de BA hacia este componente en comparación con el resto de los rodales $\left(36.78 \mathrm{Mg} \mathrm{ha}^{-1}\right)$. En cuanto a la proporción relativa de BA asignada hacia ramas (Fig. 3) se observó que el mayor porcentaje fue obtenido en el rodal de anualidad 1998 con una asignación de 17\% respecto a la BAT, disminuyendo hasta $14 \%$ en el rodal de anualidad 1982. De igual manera, el AN fue donde se encontró el mayor porcentaje de asignación de BA hacia ramas en comparación con el resto de los rodales, representando 19\% de la BAT (Fig. 3).

Por su parte, el contenido de BA asignada hacia corteza aumentó con la edad y desarrollo del rodal. Sin embargo, las anualidades 1984 y 1982, se mantuvo relativamente constante $\left(16.17 \mathrm{Mg} \mathrm{ha}^{-1}\right.$ y $16.83 \mathrm{Mg} \mathrm{ha}^{-1}$, respectivamente; Tabla 4). Por su parte, el AN presentó mayor contenido de BA que el resto de los rodales. A pesar de ello, la proporción relativa en este componente fue similar en el AN y el rodal de anualidad 1984 y 1982, representando $9 \%$ respecto a la BAT (Fig. 3 ).

El follaje fue el componente estructural con menor contenido y asignación de BA en comparación con el resto de los componentes cuantificados en el presente estudio (Tabla 3). Este componente mostró una disminución con el desarrollo del rodal. Por ejemplo, en el rodal de anualidad 1998 se observó que el contenido de BA fue mayor que en el rodal de anualidad 1995 y 1991 (3.55 Mg ha-1, 2.50 Mg ha-1 y $2.56 \mathrm{Mg} \mathrm{ha}^{-1}$, respectivamente; Tabla 3). El rodal de la anualidad 1998 representó 9\% de la BAT asignada hacia hojas, mientras que el rodal de anualidad 1995 representó 7\% (Fig. 3a). La proporción relativa de BA asignada hacia este componente, disminuyó en rodales de desarrollo intermedio, tal como ocurrió en el rodal de anualidad 1991 y 1988 que representaron 4\% de la BAT (Fig. 3a). Respecto al AN la asignación de BA en hojas fue similar a la del rodal con mayor desarrollo (anualidad 1982), representando 5\% de la BAT (Fig. 3). 


\section{DISCUSIÓN}

La comparación entre rodales de diferente edad (obtenida a partir de la aplicación de la corta de repoblación) permitió observar tendencias coincidentes con los cambios esperados para el desarrollo estructural de los rodales. Pese a que no se dispuso de repeticiones para las edades, las diferencias entre rodales pueden interpretarse como consecuencia de la diferencia de edad en función de la similitud de las condiciones climáticas, edáficas y prácticas silviculturales. De esta forma, la BAT mostró una tendencia de aumento con la etapa de desarrollo del rodal, alcanzando $78 \%$ de biomasa contenida en el AN después de 30 años de aprovechamiento. Dicho porcentaje fue solo $22 \%$ menor que el observado en el AN (Fig. 2c) con aproximadamente 80 años de edad. Lo anterior sugiere que los tratamientos aplicados en los rodales intervenidos, como parte del plan de manejo establecido para el área de estudio, han cumplido con el objetivo de aumentar la producción de BAT. El aclareo, por ejemplo, es una corta intermedia que se aplica con mayor recurrencia a nivel regional y de acuerdo a la literatura es el segundo factor en importancia para controlar la estructura, la productividad, el tamaño de los árboles y la asignación de biomasa hacia los componentes estructurales de los árboles (Arias, 2004; Rodríguez-Ortíz et al., 2012). De esta forma, el aclareo estimula el crecimiento en diámetro de los árboles y en menor medida en altura, a través del control de la densidad tanto como se requiera en cada rodal (Allen et al., 2002; Velázquez et al., 2004). Durante el período de estudio, se registró la aplicación de aclareos en dos años: 2009 y 2011 que, si bien es cierto que disminuyeron la densidad en los rodales de anualidad 1998 y 1988, los valores en el área basal $(\mathrm{AB})$ sugieren que la productividad de los rodales y la asignación de BA hacia el fuste no se observó comprometida, ya que aumentó a través del desarrollo del rodal.

La mayor asignación de BA fue hacia fuste. Lo anterior es el reflejo del aumento de la producción maderable como resultado de la aplicación de aclareos, los cuales promovieron la redistribución del espacio de crecimiento entre los árboles remanentes (Rötzer, Dieler, Mette, Mos- hammer y Pretzsch, 2010), tal y como era esperado bajo el contexto de la aplicación de esta práctica silvicultural. Al respecto, Rodríguez-Ortíz et al. (2011) encontraron que en un bosque de P. patula sometido a diferentes niveles de aclareos presentó aumentos significativos en la asignación de biomasa hacia el incremento en diámetro del fuste a cualquier nivel de aclareo, mientras que la eliminación de especies competidoras también estimularon el crecimiento de los árboles en altura. Por lo que además los aclareos permiten el alcance de las medidas comerciales adecuadas de los árboles en menor tiempo. De acuerdo con Velázquez et al. (2004), las medidas comerciales observadas para $P$. patula van de $10 \mathrm{~m}$ a $20 \mathrm{~m}$ de altura y de $25 \mathrm{~cm}$ a $50 \mathrm{~cm}$ de diámetro.

Así mismo, la asignación de BA hacia fuste varió a través del desarrollo de los rodales, lo cual está directamente relacionado con el crecimiento en altura y diámetro de los árboles y que de acuerdo con Oliver y Larson (1990) se presenta en etapas intermedias de desarrollo de un rodal y que da a lugar a un proceso de diferención de tamaño (diámetro y altura). Por ejemplo, en este estudio un rodal de 14 años después del aprovechamiento mostró $70 \%$ de asignación de BA en fuste, mientras que en el AN de aproximadamente 80 años fue de 66\% (Fig. 3). Este comportamiento podría responder a que mayores requerimientos de carbohidratos son demandados en las primeras etapas de desarrollo de un bosque ya que el incremento de biomasa en el fuste da soporte y regula la conducción de nutrimentos y agua de las raíces hacia la parte aérea de los árboles (Ryan, Binkley y Fownes, 1997). Sin embargo, a medida que aumenta la etapa de desarrollo del rodal la productividad primaria neta y las tasas de acumulación de biomasa disminuyen debido al aumento en los procesos de respiración (Tateno et al., 2009). De esta forma, desde un punto de vista funcional los aclareos podrían provocar un efecto similar al de fertilización (Smith et al., 2000; Imbert, Blanco y Castillo, 2004) es decir, con la apertura del dosel disminuye la competencia entre especies y genera un aumento en la disponibilidad y recirculación de nutrimentos. El destino de estos nutrimentos podría ser hacia los diferentes componentes estructurales de los árboles 
que dependerá de los sus requerimientos fisiológicos en cada etapa de desarrollo del rodal (Vitousek y Reiners, 1975; Imbert et al., 2004).

Otro componente importante con relación a la producción maderable, es la formación de ramas. La asignación de biomasa hacia ramas disminuyó conforme aumentó la edad después del aprovechamiento del rodal (Fig. 3). Bajo el contexto de aprovechamiento forestal, esta condición resultó favorable para el desarrollo del bosque dado que menor cantidad de ramas será igual a menor presencia de nudos en fuste, lo que determinará una mejor calidad de la madera aprovechable. Aunque $P$. patula presenta buena poda natural (Velázquez et al., 2004), en rodales con alta densidad la asignación de BA hacia ramas tendería a alterar su crecimiento, trayendo como consecuencia una disminución considerable en la altura comercial de los árboles. Por ello se recomienda que la producción de ramas sea controlada cuidadosamente durante el proceso de aprovechamiento. En este componente, la asignación de BA debe ser cuidadosamente comparable con la del AN, ya que en un área sin aprovechamiento dicha asignación podría estar influencia por la composición y distribución de especies que difiere entre coníferas y latofoliadas (Villar et al., 2004) y el crecimiento en uno y otro grupo de especies será distinto. Por ejemplo, especies latifoliadas presentan hábito de crecimiento simpódico, por lo tanto, la BA en ramas tiende a ser mayor que en coníferas. Este hecho justifica que en el AN la BA asignada hacia ramas fue mayor que la del resto de los rodales (Fig. 3a y c). Al respecto, Aquino-Ramírez et al. (2015) encontraron que en especies latifoliadas como Cupania dentata, Alchornia latifolia e Inga punctata en promedio la mayor cantidad de biomasa es obtenida en ramas (44\% a $51 \%)$ y en segundo lugar la asignación fue hacia fuste, de 33\% a $48 \%$ de la BAT.

Por su parte, el follaje que es un componente de gran importancia por ser la maquinaria fotosintética que controla directamente la productividad del ecosistema (Ryan et al., 1997; Vose y Ryan, 1994; Van y Franklin, 2000; Pretzsch, 2014), representó 4\% a 9\% de la BAT. Estos porcentajes coincidieron con otras obser- vaciones realizadas en la misma área de estudio (Figueroa et al., 2010; Soriano-Luna et al., 2015). De esta forma, la asignación de BA hacia este componente fue mayor en las primeras etapas de desarrollo de los rodales (anualidad 1998 y 1995) y se mantuvo constante en las etapas de mayor desarrollo (anualidad 1984 y 1982) incluyendo el AN. Estos resultados indicaron que, al igual que en ecosistemas sin aprovechamiento forestal, los rodales intervenidos en las primeras etapas de desarrollo la asignación de BA hacia follaje será mayor, dado que los árboles tienen mayor capacidad fisiológica para capturar y usar la energía solar, incrementando el área foliar, las tasas fotosintéticas (Ryan et al., 1997) y la producción de biomasa. Lo anterior coincide con estudios realizados en diferentes especies forestales, donde se ha encontrado que la biomasa foliar, área foliar y la estructura de la copa son factores determinantes en el crecimiento y desarrollo de los árboles (Vose y Ryan, 1994; Van y Franklin, 2000; Yu, Chambers, Tang y Barnett, 2003; Pretzsch, 2014).

Durante el aprovechamiento forestal y la aplicación de tratamientos intermedios, generalmente se generan residuos forestales compuestos principalmente por follaje, ramas, ramillas, estructuras reproductivas, frutos, corteza, entre otros (Martínez, 2014). Mucho de este material es incorporado al suelo en su mayoría aún verde y se ha encontrado que puede ser de mayor calidad nutrimental que el senescente. Al respecto, Pérez-Suárez, ArredondoMoreno, Huber-Sannwald y Vargas-Hernández, (2009) analizaron la calidad del mantillo en hojas senescentes y verdes en un bosque semiárido de Pino-Encino; encontraron que la contribución relativa de carbono, nitrógeno y fósforo por las hojas verdes a la entrada anual total fue de $10.8 \%, 12.6 \%$ y 9.4\%, respectivamente. Además, la mayor calidad representa una reincorporación más rápida (Pérez-Suárez et al., 2009) incrementando su participación en la dinámica de nutrientes del suelo de los rodales bajo aprovechamiento. Por ello, la biomasa que se incorpora al suelo a través del follaje verde, las ramas y la corteza puede representar un mecanismo importante en las entradas de nutrientes hacia el piso forestal. 
El aporte del follaje al piso forestal puede constituir entre $60 \%$ y $75 \%$ del material senescente (Imbert et al., 2004). En los bosques de P. patula el aporte es continuo, enriqueciendo significativamente el contenido de la materia orgánica, además de venir acompañado por otro tipo de especies, lo que definiría la cantidad de nutrientes transferidos al suelo y que estarán en función de la biomasa vegetal (Imbert et al., 2004). Lo anterior sugiere la importancia de realizar futuras investigaciones sobre la estimación del aporte nutrimental del follaje senescente y verde en bosques bajo aprovechamiento forestal, lo que permitiría complementar el patrón de asignación de biomasa hacia este componente.

\section{CONCLUSIONES}

La producción de biomasa aérea total aumentó a lo largo de la cronosecuencia. Sin embargo, en las primeras etapas de desarrollo del rodal no se encontraron diferencias estadísticas entre las anualidades, sino a partir de 24 años después de la cosecha. Por su parte, el rodal de mayor desarrollo (anualidad 1982) después de 30 años de haber sido aplicada la corta de repoblación, presentó $78 \%$ de la BAT de la cuantificada en el área de referencia (AN). Por lo tanto, en términos de producción de biomasa, el aprovechamiento forestal de los bosques de P. patula bajo aprovechamiento podría alcanzar niveles de biomasa aérea similares a los de un área sin manejo en un menor tiempo.

El patrón de distribución de la biomasa aérea (BA) en los diferentes componentes estructurales de los árboles presentó un orden en donde la mayor asignación se registró en el fuste. La asignación de BA hacia este componente fue favorecido por la aplicación de cortas intermedias, como los aclareos. Mientras que la asignación de BA hacia ramas disminuyó a través del desarrollo de los rodales, lo cual aumentó la calidad de la madera como resultado del control de la densidad y eliminación de especies competidoras. Por su parte, la asignación de BA hacia follaje es determinante en las primeras etapas de desarrollo del rodal.

El conocimiento sobre el patrón de asignación de biomasa hacia los diversos componentes estructurales de los árboles y su cuantificación es importante para entender la dinámica a lo largo del desarrollo estructural de los rodales bajo un régimen de aprovechamiento forestal. Esta información puede ser incorporada como base para el diseño de prácticas silvicuturales en los programas de manejo forestal y contribuir sobre la evaluación de la sustentabilidad de los bosques. El manejo forestal se plantea como una alternativa para la mitigación del cambio climático a través de la fijación y almacenamiento de bióxido de carbono.

\section{RECONOCIMIENTOS}

Esta investigación fue financiada por el Programa de Paisajes Sustentables de la Agencia para el Desarrollo Internacional de Estados Unidos de América, a través de la Oficina de Programas Internacionales del Servicio Forestal del Departamento de Agricultura, y la Northern Research Station.

\section{REFERENCIAS}

Acosta-Mireles, M., Vargas-Hernández, J., Velázquez-Martínez, A. y Etchevers-Barra J.D. (2002). Estimación de la biomasa aérea mediante el uso de relaciones alométricas en seis especies arbóreas en Oaxaca, México. Agrociencia, 36, 725-736.

Aguirre-Salado, C., Valdez-Lazalde, J. R., Ángeles-Pérez, G., De los Santos-Posadas, H., Haapanen, R. y AguirreSalado, A. I. (2009). Mapeo de carbono arbóreo aéreo en bosques manejados de pino patula en Hidalgo, México. Agrociencia, 43, 209-220.

Allen, C. D., Savage, M., Falk, D. A., Suckling, K. F., Swetnam, T. W., Schulke, T., Stacey, P. B., Morgan, P., Hoffman, M. and Klinglel, J. T. (2002). Ecological restoration of Southwestern ponderosa pine ecosystems: a broad perspective. Ecological Applications, 12 (5), 1418-1433.

Ángeles-Pérez, G. (2009). El bosque mesófilo de montaña en el estado de Hidalgo. En A. I. Monterroso-Rivas (Ed.), El bosque mesófilo de montaña en el estado de Hidalgo: perspectiva ecológica ante el cambio climático (39-54). México: Universidad Autónoma Chapingo.

Aquino-Ramírez, M., Velázquez-Martínez, A., CastellanosBolaños, J. F., De los Santos-Posadas, H. y Etchevers- 
Barra, J. D. (2015). Partición de la biomasa aérea en tres especies arbóreas tropicales. Agrociencia, 49, 299-314.

Arias, A. D. (2004). Validación del índice de densidad del rodal para el manejo de plantaciones forestales de Tectona grandis L. f. en el trópico. Kurú; Revista Forestal, 1 (1), $1-7$.

Bazzaz, F. A. (1997). Allocation of resources in plants: state of the science and critical questions. En F. A. Bazzaz y J. Grace (Eds.), Plant resource allocation (1-37). Nueva York, EUA: Academic Press.

Brandeis, T. J., Delaney, M., Parresol, B. R. y Royer, L. (2006). Development of equations for predicting Puerto Rican subtropical dry forest biomass and volume. Forest Ecology and Management, 233, 133-142.

Castelán-Lorenzo, M. y Arteaga-Martínez, B. (2009). Establecimiento de regeneración de Pinus patula Schl. et Cham. en cortas bajo el método de árboles padres. Revista Chapingo Serie Ciencias Forestales y del Ambiente, 15 (1), 49-57.

Castillo, F. J., Imbert, J. B., Blanco, J. A., Traver, C. y Puertas, F. (2003). Gestión forestal sostenible de masas de pino silvestre en el Pirineo Navarro. Ecosistemas 3. Recuperado de: http://www.aeet.org/ecosistemas/033/investigacion3.htm

Chave, J., Condit, R., Aguilar, S., Hernández, A., Lao, S. and Perez, R. (2004). Error propagation and scaling for tropical forest biomass estimates. Philosophical Transactions of the Royal Society B: Biological Sciences, 359 (1443), 409-420.

Díaz-Franco, R., Acosta-Mireles, M., Carrillo-Anzures, F., Buendía-Rodríguez, E., Flores-Ayala, E. y EtcheversBarra, J. D. (2007). Determinación de ecuaciones alométricas para estimar biomasa y carbono en Pinus patula Schl. et Cham. Madera y Bosques, 13 (1), 25-34.

Durán, M. E., Mas, J. F. y Velázquez, A. (2007). Cambios en las coberturas de vegetación y usos del suelo en regiones con manejo forestal comunitario y áreas naturales protegidas de México. En: D. Bray, L. Merino y D. Barry (Eds.), Los bosques comunitarios de México. Manejo sustentable de paisajes forestales (267-302). México D. F.: Instituto Nacional de Ecología (INE-Semarnat).
Dvorak, W. S., Hodge, G. R., Kietzka, J. E., Malan, F., Osorio, L. F. and Stanger, T. K. (2000). Pinus patula. En CAMCORE Cooperative, Central America and Mexico Resources Cooperative. Conservation and testing of tropical and subtropical forest tree species by the CAMCORE Cooperative. Raleigth, NC, EUA: Grow Graphics 2000.

Escobar, E., Maass, M., Alcocer D., J., Azpra R., E., Falcón A., L. I., Gallegos G., A. García, F. J., García-Oliva, F., Jaramillo, V., Lecuanda C., R., Magaña, V., Martínez-Yrízar, A., Muhlia V., A., Rodríguez S., R. y Zavala-Hidalgo, J. (2008). Diversidad de procesos funcionales en los ecosistemas. Vol. I: Conocimiento actual de la biodiversidad. México: Conabio.

Figueroa N., C. M., Ángeles P., G., Velázquez M., A. y de los Santos P., H. M. (2010). Estimación de la biomasa en un bosque bajo manejo de Pinus patula Schltdl. et Cham. en Zacualtipán, Hidalgo. Revista Mexicana de Ciencias Forestales, 1, 147-157.

Galicia, L., Saynes, V. y Campo, J. (2015). Biomasa aérea, biomasa subterránea y necromasa en una cronosecuencia de bosques templados con aprovechamiento forestal. Botanical Sciences, 93 (3), 473-484.

García, E. (2004). Modificaciones al sistema de clasificación climática Köppen ( $5^{\mathrm{a}}$ ed). Serie Libros. No. 6. México: Instituto de Geografía, UNAM.

Gerez-Fernández, P. y Pineda-López, M. R. (2011). Los bosques de Veracruz en el contexto de una estrategia estatal REDD+. Madera y Bosques, 17 (3), 7-27.

Gómez-Díaz, J. D., Etchevers-Barra, J. D., Monterrosos-Rivas, A. I., Campo-Alvez, J. y Tinoco-Rueda, J. A. (2011). Ecuaciones alométricas para estimar biomasa y carbonos en Quercus magnoliaefolia. Revista Chapingo Serie Ciencias Forestales y del Ambiente, 17 (2), 261-272.

Imbert, J. B., Blanco, J. A. y Castillo, F. J. (2004). Gestión forestal y ciclos de nutrientes en el marco del cambio global. En F.Valladares (Ed). Ecología del bosque mediterráneo en un mundo cambiante (479-506). Ministerio de Medio Ambiente. Madrid: EGRAF, S. A.

Jandl, R., Lindner, M., Vesterdal, L., Bauwens, B., Baritz, R., Hagedorn, F., Johnson, D. W., Minkkinen, K. y Byrne, K. 
A. (2007). How strongly can forest management influence soil carbon sequestration? Geoderma, 137, 253-268.

Law, B. E., Sun, O. J., Campell, J., Van Tuyl, S. y Thornton, P. E. (2003). Changes in carbon storage and fluxes in a chronosequence of ponderosa pine. Global Change Biology, 9, 510-524.

León, J. D., González, M. I. y Gallardo, J. F. (2011). Ciclos biogeoquímicos en bosques naturales y plantaciones de coníferas en ecosistemas de alta montaña de Colombia. Revista de Biología Tropical (International Journal of Tropical Biology and Conservation), 59 (4), 1883-1894.

Martínez C., B. E. (2014). Descomposición de la fracción foliar de Pinus patula Schltdl. \& Cham. y dinámica de la producción de hojarasca en un bosque manejado en el estado de Hidalgo. Tesis de Maestría. Colegio de Postgraduados. Postgrado Forestal. Montecillo, Texcoco, Edo. de México. Montero, G., Ruiz-Peinado, R. y Muñoz, M. (2005). Producción de biomasa y fijación de $\mathrm{CO}_{2}$ por los bosques españoles. Instituto Nacional de Investigación y Tecnología Agraria y Alimentaria. Recuperado de https:/www. researchgate.net/publication/235639682

Morales-González, E., López-Upton, J. Vargas-Hernández, J. J. Ramírez-Herrera, C. y Muñoz, A. G. (2013). Parámetros genéticos de Pinus patula en un ensayo de progenies establecido en dos altitudes. Revista Fitotecnia Mexicana, 36 (2), 155-162.

Oliver, C. D. y Larson, B. C. (1990). Forest and stand dynamics. Nueva York, EUA: Mac Graw-Hill.

Patiño, V. F. y Kageyama, P. Y. (1992). Pinus patula Schiede \& Deppe. Seedleaflet. No. 8A. Humlebaek. Dinamarca: Danida Forest Seed Centre.

Pérez-Suárez, M., Arredondo-Moreno, J. T., Huber-Sannwald, E. and Vargas-Hernández, J. J. (2009). Production and quality of senesced and green litterfall in a pine-oak forest in central-northwest Mexico. Forest Ecology and Management, 258, 1307-1315.

Pérez, C., Frangi, J., Goya, J., Luy, A. y Arturi, M. (2013). Contenido de nutrientes en las raíces finas y el mantillo de rodales de Eucalyptus grandis de diferente edad en la Mesopotamia Argentina. Bosque, 34 (3), 303-310.

Pompa-García, M. y Yerena-Yamalliel, J. I. (2014). Concentración de carbono en Pinus cembroides Zucc: fuente poten- cial de mitigación del calentamiento global. Revista Chapingo Serie Ciencias Forestales y del Ambiente, 20 (3), 169-175.

Ponce-Vargas, A., Luna-Vega, I., Alcántara-Ayala, O. y RuizJiménez, C. A. (2006). Florística del bosque mesófilo de montaña de Monte Grande, Lolotla, Hidalgo, México. Revista Mexicana de Biodiversidad, 77, 177-190.

Porter-Bolland, L., Ellis, E. A., Guariguata, M. R., RuizMallén, I., Negrete-Yankelevich, S. and Reyes-García, V. (2012). Community managed forests and forest protected areas: An assessment of their conservation effectiveness across the tropics. Forest Ecology and Management, 268, 6-17.

Pretzsch, H. (2014). Canopy space filling and tree crown morphology in mixed-species stands compared with monocultures. Forest Ecology and Management, 327, 251-264.

Rodríguez-Ortíz, G., Aldrete, A. González-Hernández, V. A., de los Santos-Posadas, H. M., Gómez-Guerrero, A. y Fierros-González, A. M. (2011). ¿Afecta los aclareos la acumulación de biomasa aérea en una plantación de Pinus patula?. Agrociencia, 45, 719-732.

Rodríguez-Ortíz, G., de los Santos-Posadas, H. M., GonzálezHernández, V. A., Aldrete, A., Gómez-Guerrero, A. y Fierros-González, A. M. (2012). Modelos de biomasa aérea y foliar en una plantación de pino de rápido crecimiento en Oaxaca. Madera y Bosques, 18 (1), 25-41.

Rötzer, T., Dieler, J., Mette, T., Moshammer, R. y Pretzsch. H. (2010). Productivity and carbon dynamics in managed Central European forests depending on site conditions and thinning regimes. Forestry, 83 (5), 483-496.

Ryan, M. G., Binkley, D. y Fownes, J. H. (1997). Age-relate decline in Forest Productivity: Pattern and Process. Advances in Ecological Research, 27, 214-262.

Rzedowski, J. (1996). Análisis preliminar de la Flora Vascular de los bosques mesófilos de montaña de México. Acta Botanica Mexicana, 35, 25-44.

SAS Institute Inc. (2009). SAS ${ }^{\odot}$ Web Report Studio 4.2; User's Guide, Cary, NC; SAS Institute Inc. 5136 p.

Smith, C. T., Lowe, A. T., Skinner, M. F., Beets, P. N., Schoenholtz, S. H. y Fang, S. (2000). Response of radiata pine forests to residue management and fertilization across a 
fertility gradient in New Zealand. Forest Ecology and Management, 138, 203-223.

Servicio Meteorológico Nacional. (2014). Normas Climatológicas. Dirección General de Geografía y Meteorología. Secretaría de Agricultura y Ganadería. México. Consulta personal en abril 2014.

Soriano-Luna, M. A., Ángeles-Pérez, G., Martínez-Trinidad, T., Plascencia-Escalante, F. O. y Razo-Zárate, R. (2015). Estimación de biomasa aérea por componente estructural en Zacualtipán, Hidalgo, México. Agrociencia, 49, 423438.

Tateno, R., Fukushima, K., Fujimaki, R., Shimamura, T., Ohgi, M., Arai, H., Ohte, N., Tokuchi, N. and Yoshioka, T. (2009). Biomass allocation and nitrogen limitation in a Cryptomeria japonica plantation chronosequence. Journal of Forest Research, 14, 276-285.

Torres-Rojo, J. M., Moreno-Sánchez, R. y Mendoza-Briseño, M. A. (2016). Sustainable forest management in Mexico. Current Forestry Reports, 2 (2), 93-105.

Van, P. R., y Franklin, J. F. (2000). Influence of canopy structure on the understory environment in tall, old-growth, conifer forests. Canadian Journal of Forest Research, 30, 1231-1245.

Vásquez, A. y Arellano, H. (2012). Estructura, biomasa aérea y carbono almacenado en los bosques del Sur y Noroccidente de Córdoba. arXiv:1208.0248. Recuperado de: http://arxiv.org/ftp/arxiv/papers/1208/1208.0248.pdf

Velázquez, M. A., Ángeles P., G., Llanderal O., T., Román J., A. R. y Reyes, H. V. (2004). Monografía de Pinus patula. México: Comisión Nacional Forestal.
Villar, R., Ruíz R., J., Quero, J. L., Poorter, H., Valladares, F. y Marañón, T. (2004). Tasas de crecimiento en especies leñosas: aspectos funcionales e implicaciones ecológicas. En F. Valladares (Ed.), Ecología del bosque mediterráneo en un mundo cambiante. Ministerio de Medio Ambiente (191-227). Madrid: EGRAF, S. A.

Vitousek, P. M. y Reiners, W. A. (1975). Ecosystem succession and nutrient retention: a hypothesis. BioScience, 25 (6), 376-381.

Von Gadow, J. M., Sánchez O., S. y Aguirre C., O. A. (2004). Manejo forestal con bases científicas. Madera y Bosques, $10(2), 3-16$.

Vose, J. M. y Ryan, M. G. (1994). Seasonal respiration of foliage, fine roots, and woody tissues in relation to growth, tissue $\mathrm{N}$, and photosynthesis. Global Change Biology, 8, 182-193.

Yu, S., Chambers, J. L., Tang, Z. y Barnett, J. P. (2003). Crown characteristics of juvenile loblolly pine 6 years after application of thinning and fertilization. Forest Ecology and Management, 180, 145-152.

Manuscrito recibido el 27 de octubre de 2014

Aceptado el 8 de octubre de 2016.

Este documento se debe citar como:

Chávez-Aguilar, G., Ángeles-Pérez, G., Pérez-Suárez, M., LópezLópez, M. A., García-Moya, E. y Wayson, C. (2016). Distribución de biomasa aérea en un bosque de Pinus patula bajo gestión forestal en Zacualtipán, Hidalgo, México. Madera y Bosques, 22 (3), 23-36. 